implications for future differential diagnoses of first-onset psychosis, potentially involving relevant auto-antibody and, specifically, anti-NMDA receptor screening. Further, plasmapheresis may be required and in some cases may even be clinically indicated before a diagnosis of NMDA receptor encephalitis is confirmed. This will have implications for hospital resources and will require close liaison between psychiatry and neurology services.

$\mathrm{N}$-methyl-D-aspartate receptor hypofunction, whether due to exposure to phencyclidine ingestion, NMDA receptor autoantibody or altered NMDA receptor trafficking, ${ }^{7,8}$ is now implicated even more strongly in schizophrenia. Future studies focusing on this area may provide clues not only to the screening and management of NMDA receptor encephalitis among firstepisode psychosis populations, but may also lead to a broader understanding of schizophrenia pathophysiology, with the potential for development of novel treatment strategies.

1 Dalmau J, Gleichman AJ, Hughes EG, Rossi JE, Peng X, Lai M, et al. AntiNMDA-receptor encephalitis: case series and analysis of the effects of antibodies. Lancet Neurol 2008; 7: 1091-8.

2 Gable MS, Gavali S, Radner A, Tilley DH, Lee B, Dyner L, et al. Anti-NMDA receptor encephalitis: report of ten cases and comparison with viral encephalitis. Eur J Clin Microbiol Infect Dis 2009; 28: 1421-9.

3 Fink M, Taylor MA. The many varieties of catatonia. Eur Arch Psychiatry Clin Neurosci 2001; 251 (suppl 1): 18-13.

4 Peralta V, Campos MS, de Jalon EG, Cuesta MJ. DSM-IV catatonia signs and criteria in first-episode, drug-naive, psychotic patients: psychometric validity and response to antipsychotic medication. Schizophr Res 2010; 118: 168-75.

5 Chapman MR, Vause HE. Anti-NMDA receptor encephalitis: diagnosis, psychiatric presentation, and treatment. Am J Psychiatry 2011; 168: 245-51.

6 Zandi MS, Irani SR, Lang B, Waters $\mathrm{P}$, Jones PB, McKenna $\mathrm{P}$, et al. Diseaserelevant autoantibodies in first episode schizophrenia. J Neurol 2011; 258 . 686-8.

7 Föcking M, Dicker P, English JA, Schubert KO, Dunn MJ, Cotter DR. Common proteomic changes in the hippocampus in schizophrenia and bipolar disorder and particular evidence for involvement of cornu ammonis regions 2 and 3 . Arch Gen Psychiatry 2011; 68: 477-88.

8 Schubert KO, Föcking M, Prehn JH, Cotter DR. Hypothesis review: are clathrin-mediated endocytosis and clathrin-dependent membrane and protein trafficking core pathophysiological processes in schizophrenia and bipolar disorder? Mol Psychiatry 2011; Oct 11, doi: 10.1038/mp.2011.123 (Epub ahead of print).

David R. Cotter, Department of Psychiatry, Royal College of Surgeons in Ireland Beaumont Hospital, Dublin 9, Ireland. Email: drcotter@rcsi.ie; Helen Barry, Department of Psychiatry, Royal College of Surgeons in Ireland, Beaumont Hospital Dublin; Daniel G. Healy, Joan Moroney, Department of Neurology, Beaumont Hospital, Dublin; Kieran C. Murphy, Department of Psychiatry, Royal College of Surgeons in Ireland, Beaumont Hospital, Dublin, Ireland

doi: $10.1192 /$ bjp.200.4.344a

\section{Occipital transcranial magnetic stimulation in dementia with Lewy bodies}

The results of Taylor et al's study ${ }^{1}$ are intriguing, shedding light on the pathogenesis of visual hallucinations in dementia with Lewy bodies.

However, I have some concerns about its methodology. The authors did not adopt the rather restrictive (and currently used) definition of phosphene threshold (i.e. the lowest stimulus intensity required to elicit phosphenes in $50 \%$ of trials), but used a much lower value (25\%) to minimise the number of participants who might not respond. Moreover, to ensure inclusion of all individuals in analyses, participants who did not report phosphenes up to $100 \%$ stimulator output were arbitrated a phosphene threshold of $101 \%$. The authors therefore assumed that not reporting phosphenes meant having a threshold above 100\% because of an insufficient magnetic field strength from the stimulator to induce phosphenes in these individuals. However, as far as I know, to date there is no evidence definitely demonstrating such an assumption.

As a matter of fact, in most published studies of phosphene thresholds a certain number of participants do not experience phosphenes even with a maximum stimulator output. There are some reasons which may (partially) explain such a phenomenon.

First, it is possible that owing to methodological difficulties in mapping phosphene thresholds over each square millimetre of the occipital skull, the correct point for stimulation may not be identified in each participant.

Second, unlike primary motor cortex, primary visual cortex (calcarine fissure) is deeply located, lying in the mid-sagittal plane, so that the magnetic field strength applied over the entire skull may be insufficient to reach and stimulate the visual cortex. Regarding this aspect, it is noteworthy to consider that Taylor et al used a figure-of-eight coil (and not a circular one), which, although it is much more selective and has a higher spatial accuracy, stimulates a smaller cortical area, ${ }^{2,3}$ and may generate, at least theoretically, a weaker electric current, resulting in a lower probability of evoking phosphenes.

Finally, as the authors stated, every millimetre the surface cortex is away from the stimulating coil, approximately an additional $3 \%$ of the maximum power output is required to induce an equivalent level of brain stimulation at the motor cortex (although no similar data on visual cortex stimulation are available in the literature). Such an aspect needs to be taken into account not only with regard to occipital cortical atrophy in affected patients compared with healthy controls, but also with regard to the fact that, because the lower portion of the visual cortex representing the upper visual field is farther from the scalp (as observed in magnetic resonance imaging), it is more difficult to elicit phosphenes with transcranial magnetic stimulation in the upper than in the lower visual field. ${ }^{4}$ Although in the study an adjusted phosphene threshold ratio was performed to account for possible group differences in atrophy, it is not clear whether other aspects (anatomical differences in skull thickness and portion of visual cortex stimulated) were considered.

In the light of the above, I think that the authors should have performed a statistical analysis of phosphene threshold including only those participants in whom phosphenes were actually induced.

1 Taylor JP, Firbank M, Barnett N, Pearce S, Livingstone A, Mosimann U, et al. Visual hallucinations in dementia with Lewy bodies: transcranial magnetic stimulation study. Br J Psychiatry 2011; 199: 492-500.

2 Cohen LG, Roth BJ, Nilsson J, Dang N, Panizza M, Bandinelli S, et al. Effects of coil design on delivery of focal magnetic stimulation. Technical considerations. Electroencephalogr Clin Neurophysiol 1990; 75: 350-7.

3 Hallett M. Transcranial magnetic stimulation and the human brain. Nature 2000; 406: 147-50.

4 Tani $N$, Hirata $M$, Motoki $Y$, Saitoh $Y$, Yanagisawa $T$, Goto $T$, et al Quantitative analysis of phosphenes induced by navigation-guided repetitive transcranial magnetic stimulation. Brain Stimul 2011; 4: 28-37.

Francesco Brigo, Department of Neurological, Neuropsychological, Morphological and Movement Sciences, Section of Clinical Neurology, University of Verona, Piazzale L.A. Scuro, 37134 Verona, Italy. Email: dr.francescobrigo@gmail.com

doi: 10.1192/bjp.200.4.345

Authors' reply: We agree that phosphene thresholds are typically defined at the $50 \%$ response rate level, although it should be recognised that the setting of a threshold is an arbitrary process. A number of our participants had thresholds near and 